\title{
Electro-magnetic fields around a drifting Kerr black hole
}

\author{
Ondřej Kopáček and Vladimír Karas \\ Astronomical Institute of the Academy of Sciences of the Czech Republic \\ email: kopacek@ig.cas.cz, vladimir.karas@cuni.cz
}

\begin{abstract}
A test field solution is constructed, describing the electromagnetic field around a Kerr black hole which is drifting in an arbitrary direction. An asymptotically uniform magnetic field is assumed with a general orientation (with respect to the rotation axis). Lines of force of electric and magnetic intensities are explored, as measured by an observer orbiting around the black hole (above the innermost stable circular orbit, ISCO) or freely falling (below the ISCO) down to the horizon. Magnetic null point is found.
\end{abstract}

Keywords. Black hole physics - magnetic fields - relativity

\section{Introduction}

Electromagnetic (EM) test field solutions of Maxwell equations in a curved spacetime play an important role in astrophysics because they show the effect of strong gravitational fields. On the other hand we can usually suppose that astrophysically relevant EM fields are weak enough, so that their influence upon background geometry may be neglected.

We are interested in the solutions describing an originally uniform magnetic field under the influence of the Kerr black hole. Since the Kerr metric is asymptotically flat, this EM field reduces to the original homogenous magnetic field in the asymptotic region. First, such a test field solution was given by Wald (1974) for the special case of perfect alignment of the asymptotically uniform magnetic field with the symmetry axis. More general solution for arbitrary orientation of the asymptotic field was given by Bičák et al. (1985). We use this solution to construct the EM field around the Kerr black hole which is drifting through the asymptotically uniform magnetic field.

\section{Lines of force}

Natural definition of the lines of force (of magnetic and electric fields), as measured by a given observer equipped with the orthonormal tetrad $e_{(\alpha)}^{\mu}$, is their identification with the lines along which magnetic/electric charge connected to this observer would accelerate due to the presence of the EM field $F_{\nu}^{\mu}$. Coordinate components of the magnetic (electric) field intensities are determined by (coordinate components of) the Lorentz force felt by the unit magnetic (electric) charge (Hanni, 1973):

$$
B^{\mu}={ }^{*} F^{\mu}{ }_{\nu} u^{\nu}, \quad E^{\mu}=F_{\nu}^{\mu} u^{\nu},
$$

where $u^{\nu}$ represents a 4 -velocity of the charge.

Tetrad components of the vector field determining desired lines of force are given as the spatial part of the projection onto $e_{(\alpha)}^{\mu}$ :

$$
B^{(i)}=B_{(i)}=e_{\mu}^{(i)} * F_{\nu}^{\mu} u^{\nu}, \quad E^{(i)}=E_{(i)}=e_{\mu}^{(i)} F_{\nu}^{\mu} u^{\nu},
$$



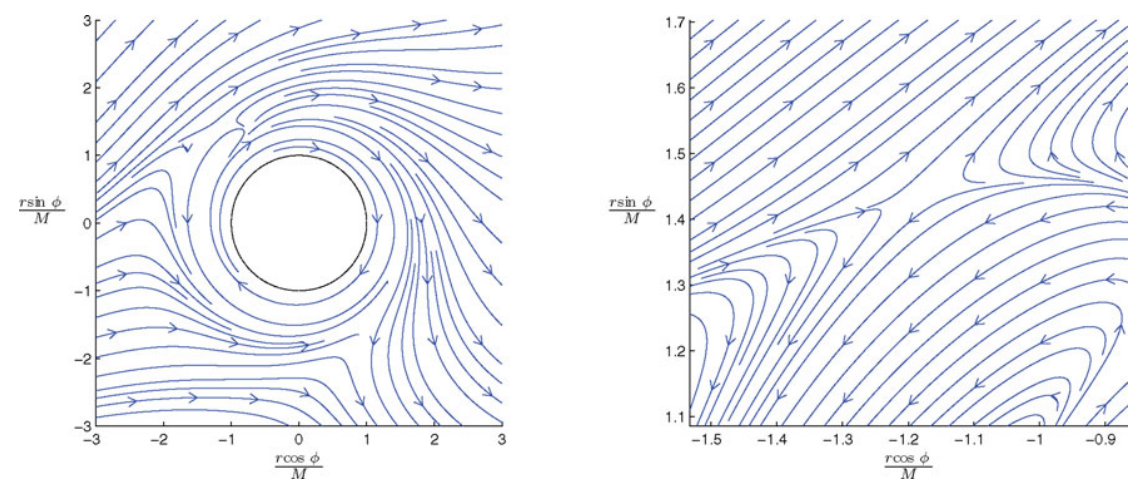

Figure 1. Counter-rotating observer around a drifting $\left(v_{x}=0.5 c\right.$ and $\left.v_{y}=-0.7 c\right)$ extreme Kerr black hole measures magnetic field (in the first panel). We notice the null point of the magnetic field (zoomed in the second panel). Original homogenous magnetic field is chosen to be parallel to the horizontal axis of these figures which show portion of the equatorial plane.

where $e_{\mu}^{(\alpha)}$ are 1-forms dual to the tetrad vectors $e_{(\alpha)}^{\mu}$. Lowering/rising spatial tetrad indices doesn't matter since the tetrad is supposed to be orthonormal: $g_{(\mu)(\nu)}=\eta_{(\mu)(\nu)}$.

Components of electromagnetic tensor $F^{\mu}{ }_{\nu}$ describing the field around a drifting Kerr black hole were derived from the non-drifting solution given by Bičák et al. (1985). General Lorentz transformation (e.g. Jackson, 1998) was used to correctly balance the asymptotic values of the components to acquire the field of desired properties.

As we are primarily interested in astrophysically relevant situations we choose the orthonormal tetrad $e_{\mu}^{(\alpha)}$ carried by the observer on the circular Keplerian orbit around the black hole. Such an orbit is specified by the values of constants of motion - by specific angular momentum $\tilde{L} \equiv u_{\phi}$ and specific energy $\tilde{E} \equiv-u_{t}$. However we don't find stable circular orbits all the way down to the event horizon. It can be shown (Bardeen et al. 1972) that there exists a radial boundary $r_{\mathrm{ms}}$ - marginally stable orbit.

For $r<r_{\mathrm{ms}}$ there are no stable circular orbits. Thus we suppose that the orbiting observer who reaches this limit performs a free fall to the black hole keeping the values of the constants of motion corresponding to the marginally stable orbit at $r_{\mathrm{ms}}$. He is falling with $\tilde{E}_{\mathrm{ms}} \equiv \tilde{E}\left(r_{\mathrm{ms}}\right)$ and $\tilde{L}_{\mathrm{ms}} \equiv \tilde{L}\left(r_{\mathrm{ms}}\right)$.

\section{Conclusions}

This work represents initial steps of our investigation of the asymptotically uniform electromagnetic test fields around a drifting Kerr black hole. Components of the electromagnetic tensor $F_{\mu \nu}$ describing such a field were derived in the terms of the nondrifting solution. The existence of the null point in Fig. 1 suggests that the magnetic reconnection may be initiated by the interplay of electromagnetic and gravitational fields. Further details can be found in Karas \& Kopáček (2008).

\section{References}

Wald, R. M. 1974, Phys. Rev. D 10, 1680

Bičák, J. \& Janiš, V. 1985, MNRAS 212, 899

Hanni, R. S. \& Ruffini, R. 1973, Phys. Rev. D 8, 3259

Jackson, J. D. 1998, Classical electrodynamics, 3rd ed, John Wiley \& Sons

Bardeen, J. M., Press, W. H., \& Teukolsky, S. A. 1972, ApJ 178, 347

Karas, V. \& Kopáček, O. 2008, CQG, in press (arXiv:0811.1772) 